\title{
Ingenolmebutat zur Behandlung der Aktinischen Keratose in der ambulanten Routineversorgung*
}

\author{
Ingenolmebutat for Treatment of Actinic Keratosis in an Outpatient Setting
}

Autoren

Institut

\author{
F. F. Gellrich, S. Gellrich
}

Dermatologische Praxis, Berlin

\section{Bibliografie}

Dol http://dx.doi.org/

$10.1055 / \mathrm{s}-0034-1377560$

Online-Publikation: 19.8 .2014

Akt Dermatol 2014; 40: 340-346

(c) Georg Thieme Verlag KC

Stuttgart · New York

ISSN 0340-2541

Korrespondenzadresse PD Dr. med. Sylke Gellrich Dermatologische Praxis Baumschulenstr. 74

12437 Berlin

sylke.gellrich@t-online.de

\section{Zusammenfassung \\ $\nabla$}

Die Aktinische Keratose ist eine häufig auftretende Erkrankung, die sich zu einem spinozellulären Karzinom entwickeln kann. 2013 wurde in Deutschland Ingenolmebutat (Picato) zur Behandlung der Aktinischen Keratose neu zugelassen. Diese Beobachtung analysiert, ob die Anwendung von Ingenolmebutat in der ambulanten Routineversorgung im Vergleich zu bereits etablierten Behandlungsmethoden, geeignet ist.

Im Rahmen der ambulanten Routineversorgung wurden alle behandlungspflichtigen Patienten mit Aktinischen Keratosen in einer dermatologischen Praxis im Zeitraum vom 31.1.2013 bis zum 14.5.2013 mit Ingenolmebutat nach Anwendungsvorschrift behandelt. Vor Beginn der Therapie, nach 8 Wochen und nach 12 Wochen wurden die Läsionen fotografisch dokumentiert sowie die subjektiven Beschwerden der Patienten erfragt. Die Fläche und Anzahl der Läsionen wurden ausgewertet. Die Ausprägung von Erythemen, Schuppung, Krustenbildung, Ödembildung, Bläschenbildung, Erosionen und Ulzerationen wurde in Anlehnung an die Zulassungsstudie von Ingenolmebutat im Local-Skin-Response-Score zusammengefasst. Zusätzlich wurden die subjektiven Beschwerden der Patienten in einer Skala von 0 bis 10 quantifiziert.

Von 62 behandelten Patienten erhielten 59 Patienten 3 Behandlungen mit Ingenolmebutat. $58 \mathrm{~Pa}-$

\section{Einleitung}

Aufgrund höherer Lebenserwartung, einem vermehrten Sonnen- und UV-Konsum und der besseren Wahrnehmung der Erkrankung durch Patienten und Ärzte werden Aktinische Keratosen häufig diagnostiziert. Es handelt sich aus histologischer

\footnotetext{
* vorgetragen auf dem 23. Deutschen Hautkrebskongress/
} ADO-Jahrestagung, 26.-28.9.2013, Essen tienten erschienen nach 12 Wochen zur Nachbeobachtung. In 51,7\% (30 Patienten) der Behandlungen konnte eine komplette Remission, in 3,4\% (2 Patienten) eine partielle Remission (Reduktion der Fläche um mehr als 75\%), in 32,8\% (19 Patienten) ein unverändertes Krankheitsbild (Reduktion der Fläche um weniger als 75\%) und in 10,3\% (6 Patienten) ein progressiver Krankheitsverlauf ( $\mathrm{Zu}-$ nahme der Fläche um mehr als 5\%) beobachtet werden. Bei einem Patienten (1,7\%) ist nach 12 Wochen ein Rezidiv aufgetreten. Bei einigen Patienten blieb 12 Wochen nach Beginn der Therapie lediglich ein dezentes Resterythem zurück. Definiert man diese Läsionen als abgeheilt, so erreichten $67,2 \%$ der Patienten eine komplette Remission. Häufig kann von Zulassungsstudien nicht auf die Praxistauglichkeit eines Medikamentes geschlossen werden. Die Studienpopulation unterscheidet sich vom Patientenklientel oft in der Altersverteilung, der Morbidität, der Compliance sowie anderer Merkmale, die auf die Therapie einen entscheidenden Einfluss haben. Diese rückwirkende Beobachtung bestätigt die Wirksamkeit von Ingenolmebutat in der ambulanten Routineversorgung. Der beobachtete Effekt der Therapie ähnelt dem der Zulassungsstudie sowie anderer Therapieoptionen. Da die Behandlung nur 3 Anwendungen verlangt und die Patienten nur wenige Beschwerden angeben, stellt die Therapie mit Ingenolmebutat eine angenehme und effektive Alternative zur Behandlung der Aktinischen Keratose dar.

Sicht um initiale Tumore epithelialer Herkunft, die als Carcinoma in situ klassifiziert werden [1]. In Ländern mit hoher UV-Belastung kommen Aktinische Keratosen gehäuft vor [2,3]. Während die Prävalenz der Aktinischen Keratosen in Großbritannien $15 \%$ bei Männern und $6 \%$ bei Frauen beträgt [4], beläuft sie sich in Australien bei der hellhäutigen Bevölkerung in der Altersgruppe von 30 bis 70 Jahren auf $55 \%$ bei Männern und $37 \%$ bei 

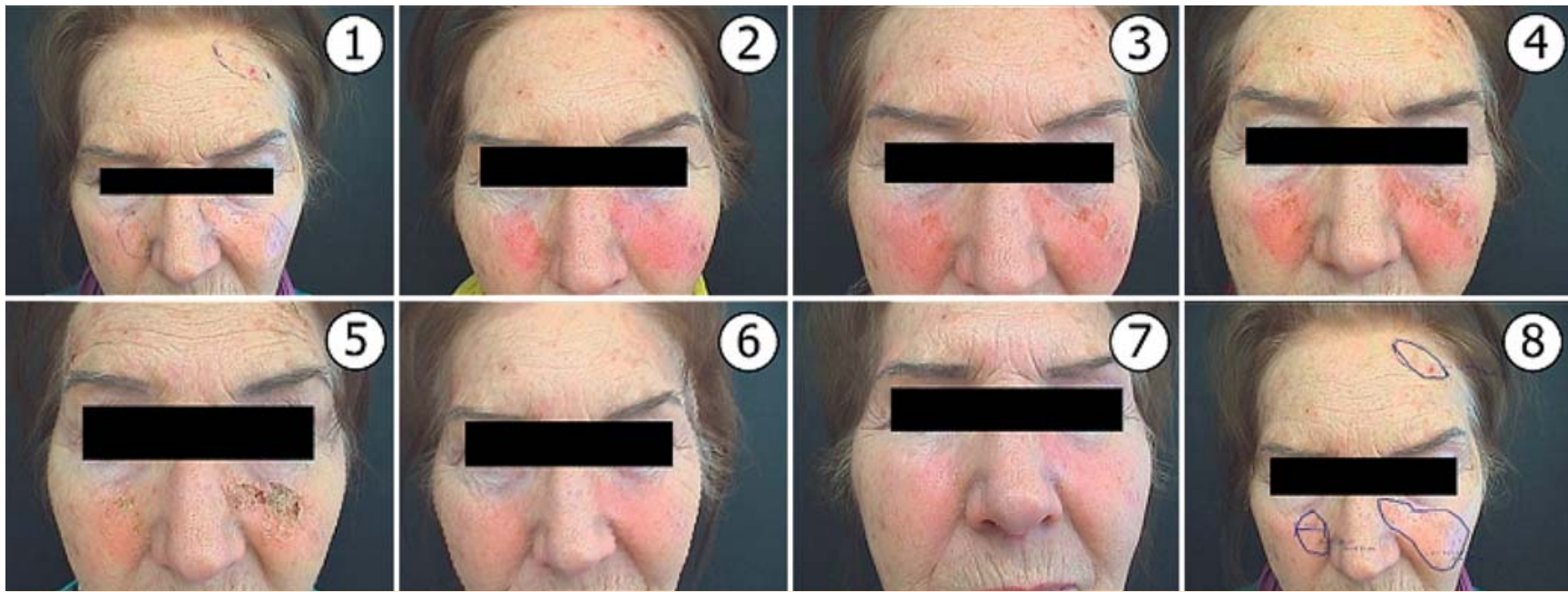

Abb.1 Abgebildet ist die Fotodokumentation einer Patientin im Verlauf der Therapie und Nachbeobachtungszeit. Zu folgenden Zeitpunkten wurde die Fotodokumentation erhoben: Bild 1: Zeitpunkt vor der Therapie, Bild 2: 1. Tag nach Therapiebeginn, Bild 3: 2. Tag nach Therapiebeginn, Bild 4: 3.Tag nach Therapiebeginn, Bild 5: 7. Tag nach Therapiebeginn, Bild 6: 4 Wochen nach Therapiebeginn, Bild 7: 8 Wochen nach Therapiebeginn, Bild 8: 12 Wochen nach Therapiebeginn.

Frauen. Im Mittel beträgt die Prävalenz 15\% bei Männern und 7\% bei Frauen [5].

Immunsupprimierte Patienten haben eine erhöhte Wahrscheinlichkeit, ein kutanes spinozelluläres Karzinom (SCC) zu entwickeln. $40 \%$ aller transplantierten Patienten entwickeln innerhalb von 5 Jahren nach der Transplantation Aktinische Keratosen, Plattenepithelkarzinome oder Basalzellkarzinome [6]. Das Risiko der Progression von Aktinischen Keratosen zu einem SCC wird mit $0,025 \%$ bis $16 \%$ angegeben. Immunsuprimierte Patienten haben ein erhöhtes Risiko (30\%), ein Plattenepithelkarzinom zu entwickeln [6] [7].

Die Hauptursache der Aktinischen Keratosen liegt in der kumulativen Dosis von ultravioletter Strahlung (UV) auf der Haut. Aus diesem Grund entstehen Aktinische Keratosen gehäuft an besonders sonnenexponierten Körperregionen. UVB-Strahlung kann durch Bildung von beispielsweise Pyrimidin-Dimeren Schäden an der menschlichen DNA verursachen. Entstehen diese Schäden im Bereich des p-53-Tumorsupressor-Gens, so erhalten jene Zellen einen Selektionsvorteil und können unkontrolliert Zellklone bilden. Findet eine solche Mutation in einem Keratinozyten statt, so kann daraus eine Aktinische Keratose entstehen [8].

Durch die Pathogenese ist die Zunahme der Aktinischen Keratosen im Alter zu erklären. In der oben zitierten Studie wurde die Aktinische Keratose in Großbritannien über einem Alter von 70 Jahren bei 34\% der Männer und 18\% der Frauen diagnostiziert.

Des Weiteren stellen heller Hauttyp, häufig berufs- und freizeitbedingte Sonnenexposition, schwere Sonnenbrände in der Kindheit, Immunsuppression und genetische Erkrankungen in der DNA-Reparatur weitere Risikofaktoren für die Aktinischen Keratosen dar [9]. Gerade das geschlechtsabhängige unterschiedliche Verhalten in den eben genannten Punkten gilt als Hauptursache für den hohen Unterschied in der Prävalenz zwischen Männern und Frauen [10].

Da das SCC der Haut metastasieren kann, ist die Behandlung seiner initialen Vorstufe, überwiegend der Aktinischen Keratosen, indiziert.

Derzeit gibt es viele Behandlungsmethoden, die jedoch unterschiedlich gut evaluiert sind. Entsprechend der deutschen Leitlinien für Aktinische Keratosen (AWMF) sind Lokaltherapeutika wie Imiquimod, Diclofenac/Hyaluronsäure, 5-Fluorouracil und kürzlich auch Ingenolmebutat in Deutschland zugelassen. Weitere Therapien zur Behandlung der Aktinischen Keratosen, wie z.B. die Kryotherapie und die fotodynamische Therapie, werden häufig angewendet [11].

Die Neuzulassung von Medikamenten und Verfahren erfolgt nach Maßgabe der jeweiligen Studienergebnisse. Untersuchungen über die Behandlung der Patienten unter ambulanten Routinebedingungen nach dem Zulassungsstatus gibt es derzeit nicht. Da Ingenolmebutat in Deutschland seit Januar 2013 indikationsgerecht zur Therapie der Aktinischen Keratosen angewendet werden kann, wurden die ersten 60 Anwendungen in einer dermatologischen Praxis in Berlin fotografisch und schriftlich dokumentiert und retrospektiv hinsichtlich der Anwendbarkeit, der Wirkung und der Nebenwirkungen analysiert.

\section{Material und Methoden \\ $\nabla$}

Im Rahmen der ambulanten Routineversorgung wurden alle behandlungspflichtigen Patienten mit Aktinischen Keratosen in einer dermatologischen Praxis im Zeitraum vom 31.1.2013 bis zum 14.5.2013 mit Ingenolmebutat behandelt: im Kopf-HalsBereich mit $150 \mu \mathrm{g} / \mathrm{g}$ Gel, an allen anderen Körperarealen mit $500 \mu \mathrm{g} / \mathrm{g}$ Gel. Die Auftragung des Medikamentes erfolgte durch das Praxispersonal über einen Zeitraum von 3 bzw. am Körper von 2 Tagen, wobei pro Anwendung entsprechend der pharmakologischen Konfektion 0,47g Gel verwendet wurden. Die Anwendungsvorschrift für Ingenolmebutat beschreibt das Auftragen einer Tube Ingenolmebutat auf einer Fläche von $25 \mathrm{~cm}^{2}$. Bei Behandlung kleinerer Flächen wurde ein Teil des Tubeninhalts verworfen, um die Konzentration pro Fläche konstant zu halten. Aufgrund der sehr kleinen und unhandlichen Tuben konnte die entsprechende Menge nur grob abgeschätzt werden. Um klinische Erfahrungen über ein in Deutschland neu zugelassenes Medikament unter Routinebedingungen zu erlangen, wurden die Verläufe dokumentiert und retrospektiv ausgewertet. Die Behandlungen und die darauffolgenden Reaktionen wurden zu verschiedenen Zeitpunkten fotografisch und anhand eines jeweils erhobenen Scores sowie durch Befragung der Patienten zu folgenden Zeitpunkten erfasst: Tag 0: Tag der ersten Behandlung, 
Tag 1, Tag 2, Tag 3/4, Tag 6/7, Tag 35 (4 Wochen), Tag 63 (8 Wochen), Tag 91 (12 Wochen) ( $\bullet$ Abb. 1$)$.

Vor Behandlung und nach 8 und 12 Wochen wurden die Fläche und die Anzahl der Läsionen fotografisch beurteilt und berechnet. Zu anderen Zeitpunkten war dies nicht möglich, da die Entzündungsreaktion eine entsprechende Messung nicht zuließ.

Der Intensitätsscore (IS) bezog sich auf die 6 Parameter Erythem, Schuppung, Krustenbildung, Ödembildung, Bläschenbildung, Erosionen und Ulzerationen in Anlehnung an die Zulassungsstudie von Ingenolmebutat [12]. Dabei wurde die Intensität jeweils von 0 bis 4 durch den behandelnden Arzt evaluiert. Die Summe der einzelnen Punkte (minimal 0 bis maximal 24 Punkte) der sechs Merkmale stellt den Local-Skin-Response-Score (LSRScore) dar. Für die Einteilung der oben genannten Merkmale wurden die Fototafeln aus der Zulassungsstudie benutzt. Somit lässt sich ein nachvollziehbarer Vergleich zwischen der Zulassungsstudie und dieser Anwendungsbeobachtung anstellen.

Die subjektiven Beschwerden (BS) Juckreiz, Brennen, Schmerzen und Spannungsgefühl wurden jeweils auf einer Skala von 1 bis 10 bewertet. Im Rahmen der ambulanten Betreuung wurden diese Beschwerden der Patienten abgefragt.

\section{Ergebnisse}

$\nabla$

Insgesamt wurden 62 Patienten kaukasischer Rasse mit Hauttyp (HT) I, II oder III (HTI = 4, HT I bis II = 7, HT II = 37, HT II bis III = 4), davon 35 Männer und 27 Frauen über einen Zeitraum von 3 Tagen behandelt und 91 Tage nachbeobachtet. Aufgrund sehr starker Lokalreaktionen wurde die Behandlung bei 2 Patienten nach dem ersten Behandlungstag und bei einem Patient nach dem zweiten Behandlungstag abgebrochen. 59 Patienten haben die 3 Anwendungen mit Ingenolmebutat entsprechend der Behandlungsvorschrift erhalten.

Nachbeobachtet wurden am Tag 762 Patienten, nach 4 Wochen 59 Patienten, nach 8 Wochen 52 Patienten und nach 12 Wochen 58 Patienten.

Das Durchschnittsalter aller Patienten beträgt 74,5 (standard deviation 7,4) Jahre. Bei den Männern liegt es mit 75,4 (SD 7,5) Jahren um durchschnittlich 2 Jahre höher als bei den Frauen mit 73,4 (SD 7,3) Jahren.

28 der 63 behandelten Patienten erhielten eine Vortherapie ( $8 \times$ Imiquimod, $4 \times$ Diclofenac/Hyaluronsäure, $16 \times$ Diclofenac/Hyaluronsäure und Imiquimod. Bei allen vorbehandelten Patienten endete die letzte Behandlung spätestens 8 Wochen vor Beginn der Therapie mit Ingenolmebutat.

Durchschnittlich manifestierten sich 4,2 Aktinische Keratosen pro Patient, (Minimum=1, Maximum=16). Die Verteilung der Läsionen zeigte sich folgendermaßen: Stirn $=21$, Nase $=21$, Jochbeine $=13$, Oberkopf $=13$, Wange $=8$, Schläfe $=6$. Außerhalb des Gesichtes wurden behandelt: Hand =3, Fuß $=1$, Rücken =3, Dekolleté $=2$, Hals $=1(\bullet$ Abb.2).

Im Mittel wurden pro Patient 4,2 (SD 2,9) Läsionen und eine Fläche von $11,4 \mathrm{~cm}^{2}\left(\mathrm{SD} 15,5 \mathrm{~cm}^{2}\right)$ behandelt. Nach einem Zeitraum von 12 Wochen sind 58 Patienten zur Nachbeobachtung erschienen.

In 51,7\% (30 Patienten) der Behandlungen konnte eine komplette Remission, in 3,4\% (2 Patienten) eine partielle Remission (Reduktion der Fläche um mehr als 75\%), in 32,8\% (19 Patienten) ein unverändertes Krankheitsbild (Reduktion der Fläche um weniger als $75 \%$ ) und in 10,3\% (6 Patienten) ein progressiver Krankheitsverlauf (Zunahme der Fläche um mehr als $5 \%$ ) beobachtet wer-

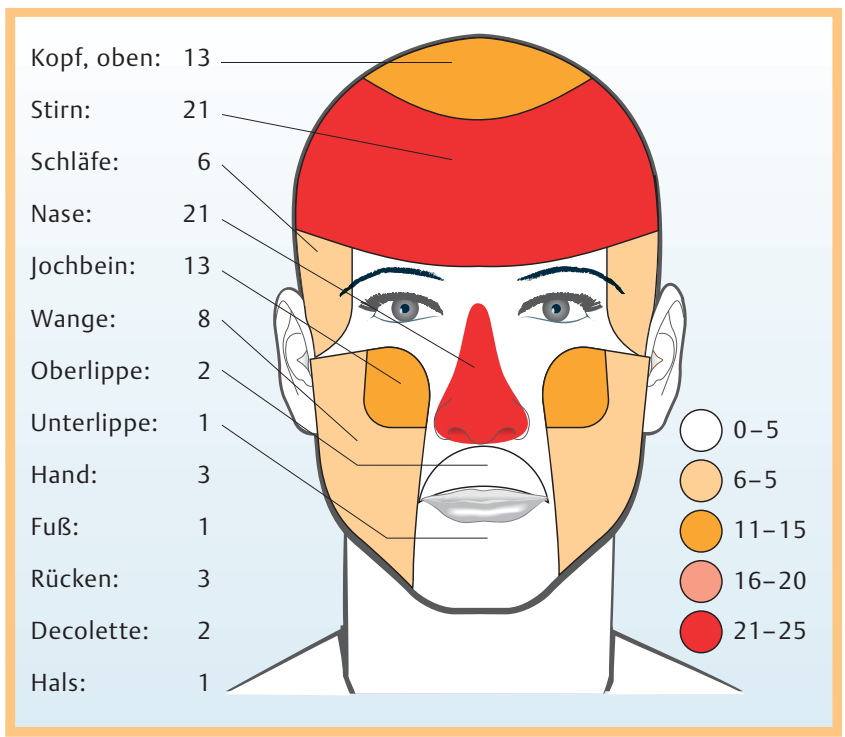

Abb.2 In dieser Grafik ist die Anzahl der Läsionen innerhalb verschiedener Areale des Kopfes abgebildet. Die farbliche Kodierung beschreibt die Häufigkeitsverteilung.

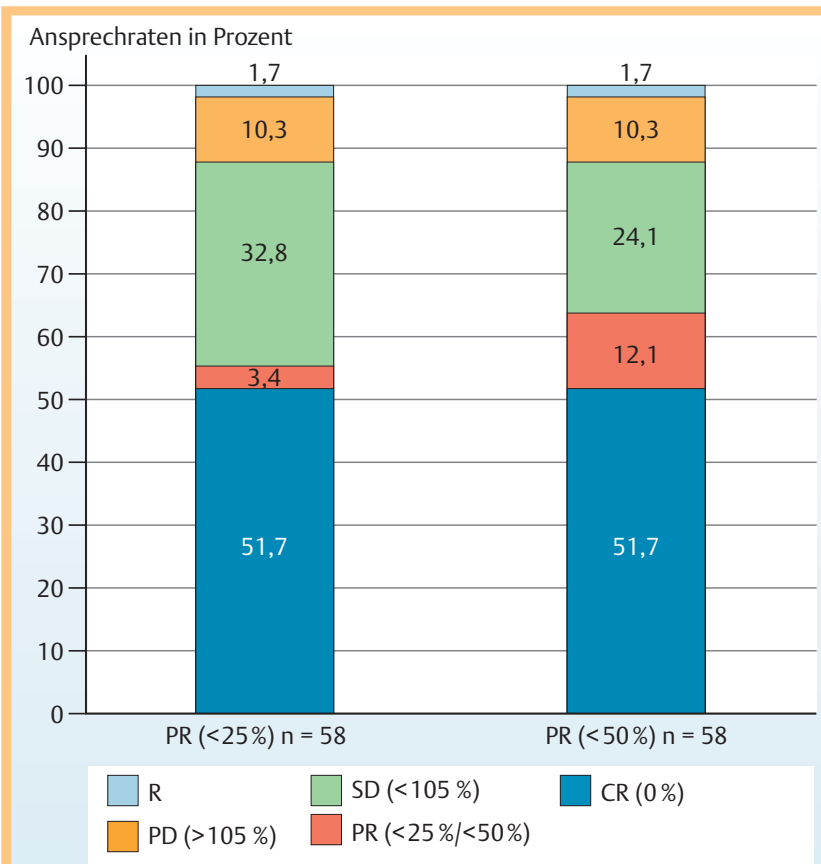

Abb.3 Nach der relativen Entwicklung der Flächen, 12 Wochen nach Beginn der Therapie, wurden die Patienten den folgenden Kategorien zugeordnet, um den Therapieerfolg zu analysieren.

CR: komplette Remission ( $0 \%$ der Ausgangsfläche); R: partielle Remission (Reduktion der Ausgangsfläche um $>75 \%$ in der 1 . Säule, um $>50 \%$ in der 2. Säule); SD: stable disease (75\% bis $105 \%$ der Ausgangsfläche); PD: progressive disease (mehr als 105\% der Ausgangsfläche); R: Rezidiv (abgeheilter Befund, der innerhalb von 12 Wochen erneut aufgetreten ist).

den. Bei einem Patienten (1,7\%) ist nach 12 Wochen ein Rezidiv aufgetreten $(\bullet$ Abb. 3$)$.

Da die Definition für die partielle Remission nicht einheitlich geregelt ist, wurden die Daten aufgrund von 2 verschiedenen Schnittpunkten dargestellt. Je nach Setzen der Grenze kann die partielle Remission mit 12,1\% (Reduktion der Ausgangsfläche 


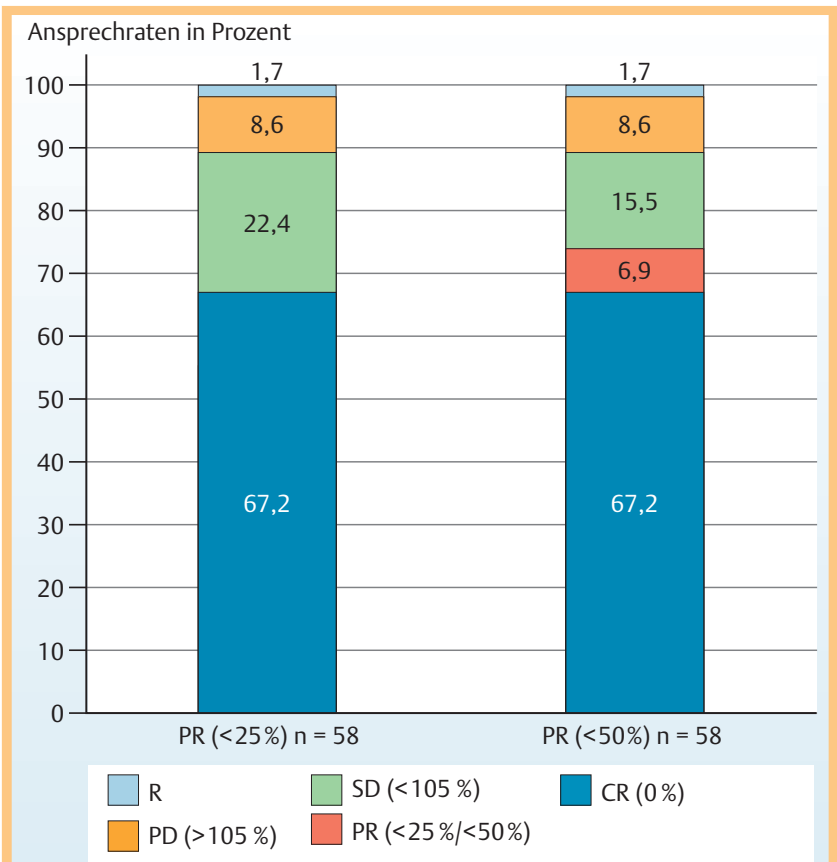

Abb.4 Nach der relativen Entwicklung der Flächen, 12 Wochen nach Beginn der Therapie, wurden die Patienten den folgenden Kategorien zugeordnet, um den Therapieerfolg zu analysieren. Ansprechraten ohne Berücksichtigung einer Rötung entsprechend LSR-Score 1.

CR: komplette Remission ( $0 \%$ der Ausgangsfläche); PR: partielle Remission (Reduktion der Ausgangsfläche um $>75 \%$ in der 1 . Säule, um $>50 \%$ in der 2. Säule); SD: stable disease (75\% bis $105 \%$ der Ausgangsfläche); PD: progressive disease (mehr als 105\% der Ausgangsfläche); R: Rezidiv (abgeheilter Befund, der innerhalb von 12 Wochen erneut aufgetreten ist).

um > 50\%) oder 3,4\% (Reduktion der Ausgangsfläche um > 75\%) angegeben werden ( Abb.3).

Es gab einige Patienten, welche nach 12 Wochen lediglich ein dezentes Resterythem (LSR 1) und sonst keine weiteren Entzündungszeichen aufwiesen. Definiert man die Aktinischen Keratosen dieser Patienten als vollständig abgeheilt, so zeigen sich noch höhere Raten der kompletten $(67,2 \%)$ und partiellen Remission ( Abb.4). Da Biopsien bei der Behandlung von Aktinischen Keratosen laut Leitlinie nicht vorgesehen sind, können auch nur die klinischen Merkmale bei der Bewertung der Abheilung herangezogen werden.

Entsprechend den klinischen Studien wurde versucht die Reduktion der Läsionenanzahl der Aktinischen Keratosen zu beurteilen, was jedoch aufgrund der Entzündungsreaktion nicht eindeutig auswertbar war.

Die Darstellung des Therapieerfolges in Abhängigkeit vom Geschlecht zeigt bei den weiblichen Patienten ein besseres Therapieansprechen. Bei 42\% (14) der männlichen Patienten und bei 64\% (16) der weiblichen Patienten konnte nach 12 Wochen eine komplette Remission, bei 6\% (2) der männlichen und keinem weiblichen Patienten eine partielle Remission (Reduktion der Ausgangsfläche um $>75 \%$ ) erzielt werden. Bei 33\% (11) der männlichen Patienten und 32\% (8) der weiblichen Patienten zeigte sich keine relevante Veränderung und bei $15 \%$ (5) der männlichen Patienten sowie 4\% (1) der weiblichen Patienten vergrößerten sich die Läsionen ( $\bullet$ Abb.5).

Behandlungen am Kopf zeigten mit 52\% kompletten Remissionen, 3\% partiellen Remissionen, 33\% unveränderten Krankheitsbildern und 10\% progressiven Krankheitsverläufen einen größe-

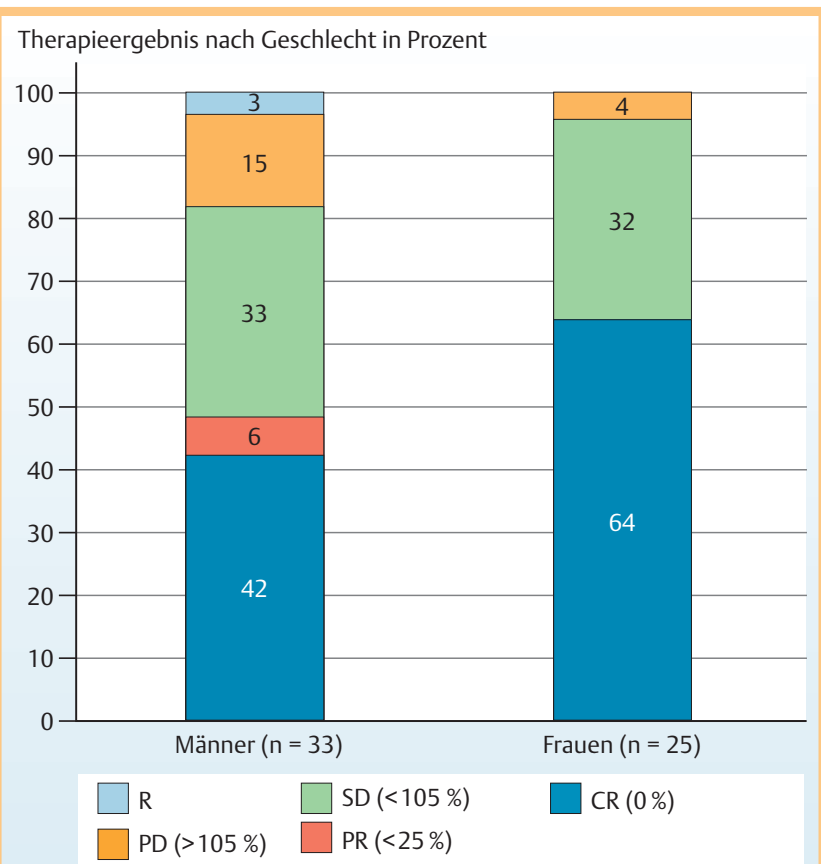

Abb.5 Ergebnis der Therapie nach 12 Wochen abhängig vom Geschlecht. CR: komplette Remission (0\% der Ausgangsfläche); PR: partielle Remission (Reduktion der Ausgangsfläche um > 75\%); SD: stable disease (25\% bis $105 \%$ der Ausgangsfläche); PD: progressive disease (mehr als 105\% der Ausgangsfläche); R: Rezidiv (abgeheilter Befund, der innerhalb von 12 Wochen erneut aufgetreten ist).

ren Therapieerfolg als Behandlungen am Körper mit 38\% kompletten Remissionen, keiner partiellen Remission, 63\% unveränderten Krankheitsbildern und keinem progressiven Krankheitsverlauf $(\bullet$ Abb.6).

Vergleicht man die am häufigsten befallenen Lokalisationen am Kopf bezüglich des Therapieerfolgs, so zeigt die Therapie im Gesicht (komplette Remissionen auf Wange in 63\%, auf Jochbein in $58 \%$, auf Nase in 55\%) einen höheren Therapieerfolg als auf dem Kopf (komplette Remissionen auf Kopfhaut in $46 \%$ und auf Stirn in $45 \%$ ) ( Abb.7).

Durchschnittlich wurden 4,3 (SD 2,9) Läsionen zum Beginn der Therapie behandelt. 12 Wochen nach Therapiebeginn konnten im Mittel 1,1 (SD 2,1) Läsionen pro Patient gezählt werden. Dies ergibt eine Reduktion der Anzahl um 74\%. 37 Patienten (59\%) haben zu diesem Zeitpunkt keine Läsionen mehr aufgewiesen. Bei 4 Patienten (6\%) konnte die Anzahl der Läsionen um mehr als 75\% reduziert werden. 2 Patienten (35\%) zeigten eine Minderung der Anzahl der Läsionen um weniger als 75 und bei keinem Patienten nahm die Anzahl der Läsionen zu ( $\bullet$ Abb.8).

Um das Ausmaß der Reaktion der Haut auf die Behandlung mit Ingenolmebutat $\mathrm{zu}$ bewerten, wurde der bereits vorgestellte LSR-Score angewendet.

Von Tag 1 bis Tag 4 nimmt die Intensität (IS) der klinischen Befunde Erythem (IS 3,8), Schwellung (IS 2,5), Bläschen/Pusteln (IS 1,5) und Erosion/Ulzera (IS 0,8) stark zu. Krusten (IS 2,0) und Schuppung (IS 2,8) bleiben bis Tag 7 im Maximum. Nach 4 Wochen hat sich die therapiebedingte Lokalreaktion komplett zurückgebildet ( Abb.9).

Um das subjektive Empfinden der Therapie durch die Patienten zu ermitteln, wurden die Patienten nach Schmerzen, Juckreiz, Brennen und Spannungsgefühl befragt und die Intensität der jeweiligen Qualität in einer Skala (BS) von 0 bis 10 eingeordnet, 


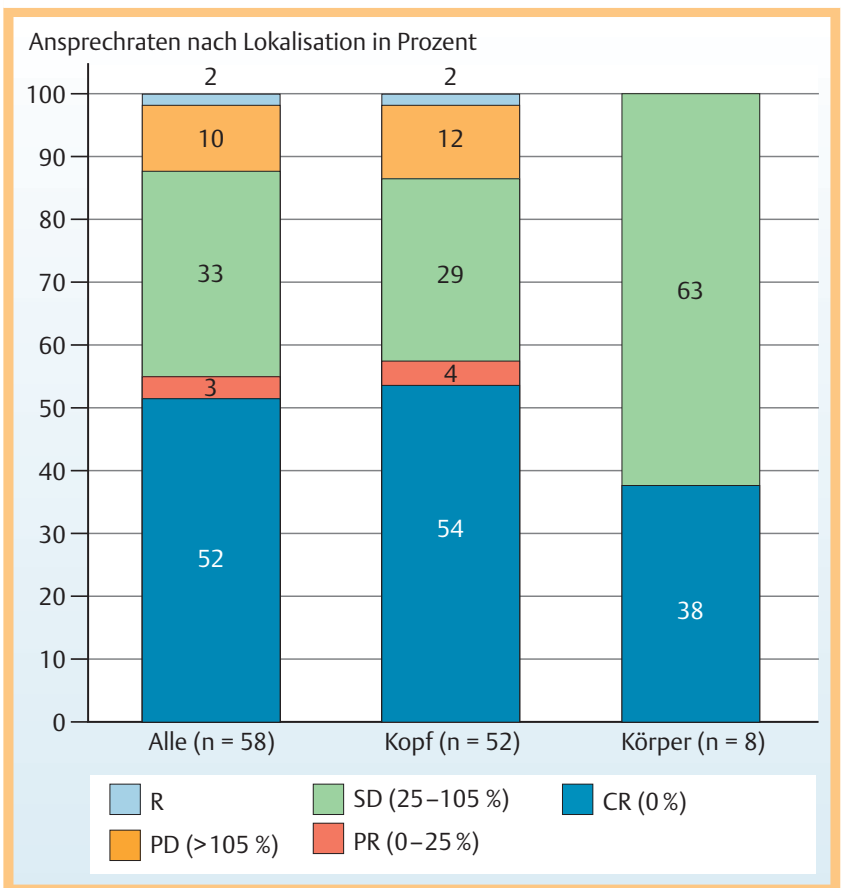

Abb. 6 Therapieerfolg nach 12 Wochen abhängig von der Lokalisation auf dem Kopf oder dem Körper.

CR: komplette Remission ( $0 \%$ der Ausgangsfläche); PR: partielle Remission ( $0 \%$ bis $25 \%$ der Ausgangsfläche); SD: stable disease ( $25 \%$ bis $105 \%$ der Ausgangsfläche); PD: progressive disease (mehr als 105\% der Ausgangsfläche); R: Rezidiv (abgeheilter Befund, der innerhalb von 12 Wochen erneut aufgetreten ist).

wobei 0 für nicht Wahrnehmen und 10 für die stärkste Ausprägung des Merkmals steht. Die Parameter erreichten folgende Maxima: Juckreiz BS 1,6 und Brennen BS 2,0 nach 3 Tagen, Schmerz BS 1,5 und Spannungsgefühl BS 2,6 nach 4 Tagen $(\odot$ Abb. 10).

\section{Diskussion}

Um die Zulassung eines Medikamentes zu erreichen, werden die Wirksamkeit, die Nebenwirkungen, die Sicherheit und ggf. der Vergleich mit anderen Medikamenten unter Studienbedingungen beurteilt. Ob das Medikament praxistauglich ist und breit angewendet werden kann oder ob die Compliance der Patienten ausreichend ist, wird nur selten erfasst.

Für die Behandlung der Aktinischen Keratosen existieren bereits mehrere etablierte Therapieverfahren. $\mathrm{Zu}$ ihnen gehören medikamentöse Möglichkeiten wie 5-Fluorouracil-Creme 5\%, Imiquimod-Creme 3,75\% und 5\% oder Diclofenac-Gel 3\%/Hyaluronsäure $2,5 \%$ Gel sowie physikalische Therapieverfahren, wie die Kryotherapie oder Ablationsverfahren [11].

Eine Studie zur Effektivität der Behandlung der Aktinischen Keratosen mit 5-Fluorouracil 5\% Creme untersuchte die Anzahl der kompletten Remissionen in Abhängigkeit von verschieden langen Behandlungszeiträumen. Mit einer Behandlungsdauer von einer Woche konnte in 26,3\% der Fälle eine komplette Remission erzielt werden, mit einer 2 Wochen andauernden Behandlung waren es $19,5 \%$ und eine vier wöchige Behandlung führte zu 47,5\% kompletten Remissionen [13].

Eine weitere Studie zur Wirkung von Imiquimod 5\% auf Aktinische Keratosen berichtet von 45,1\% kompletten Remissionen, im Gegensatz zur Kontrollgruppe mit 3,2\% [14].

Diclofenac-/Hyaluronsäure-Gel dagegen zeigt in 64,7\% eine Reduktion der befallenen Fläche (Kontrollgruppe 34,3\%) und in 9,3\% der Fälle eine komplette Remission [15].

Im März 2012 wurde die Zulassungsstudie zu Ingenolmebutat publiziert. Am Tag 57 nach Therapiebeginn wurde der Behandlungserfolg ausgewertet. Im Gesicht (Ingenolmebutat 0,015\%) konnte bei $42,2 \%$ der Patienten eine komplette Remission erreicht werden. Diese Patienten wurden weitere 12 Monate nachbeobachtet. 87,2\% der Patienten mit einer kompletten Remission blieben nach dieser Zeit rezidiv frei [12].

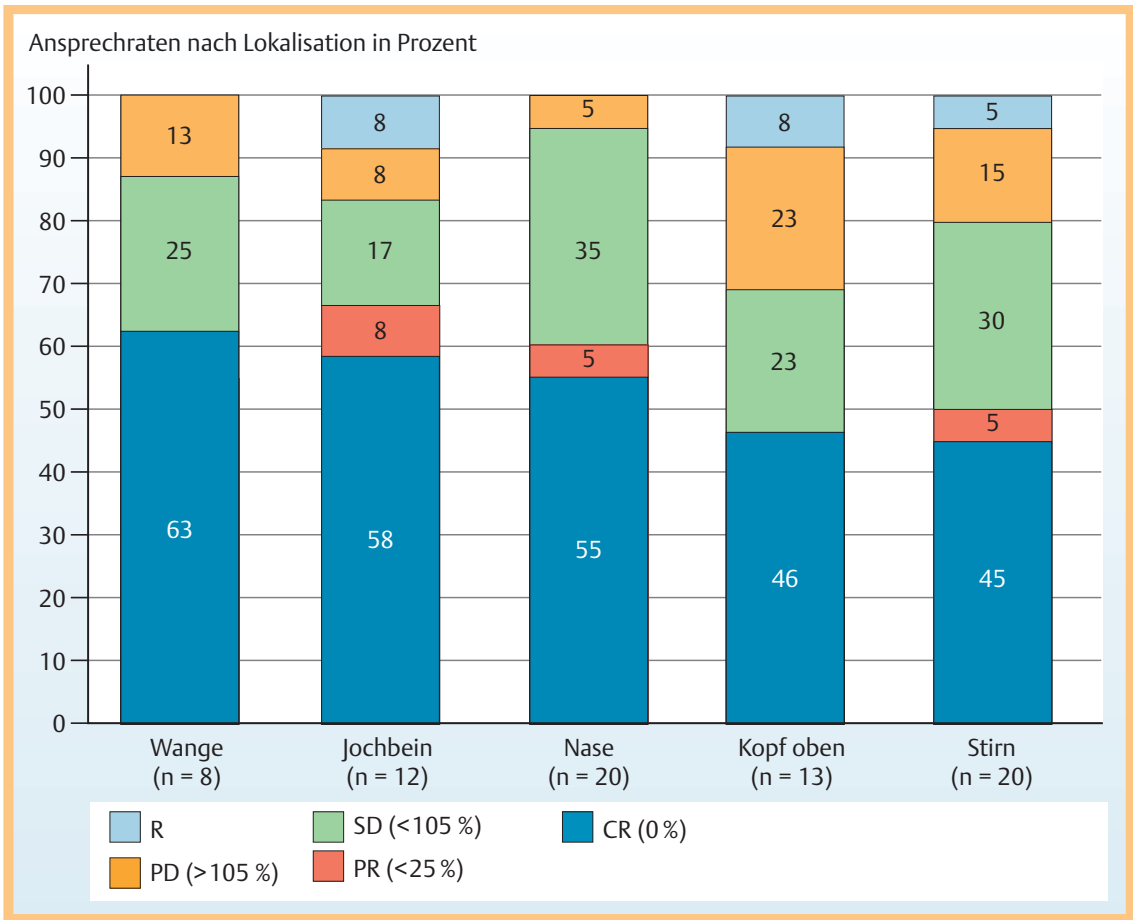

Abb. 7 Therapieerfolg nach 12 Wochen abhängig von der Lokalisation auf dem Kopf.

CR: komplette Remission ( $0 \%$ der Ausgangsfläche); PR: partielle Remission ( $0 \%$ bis $25 \%$ der Ausgangsfläche); SD: stable disease ( $25 \%$ bis $105 \%$ der Ausgangsfläche); PD: progressive disease (mehr als 105\% der Ausgangsfläche); R: Rezidiv (abgeheilter Befund, der innerhalb von 12 Wochen erneut aufgetreten ist). 


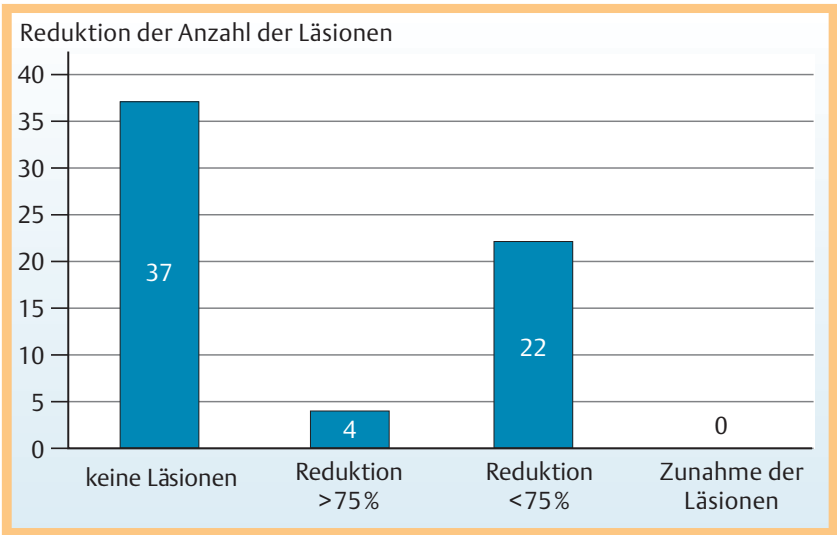

Abb. 8 Die Anzahl der Läsionen wurde vor und 12 Wochen nach Beginn der Therapie gezählt. Zu den anderen Zeitpunkten war dies nicht möglich, da die Entzündungsreaktion eine entsprechende Messung nicht zuließ. In diesem Diagramm ist die Anzahl der Patienten aufgetragen, bei denen 12 Wochen nach Beginn der Therapie keine Läsionen sichtbar waren (37), sich die Anzahl der Läsionen um mehr als 75\% verringert hat (4), sich die Anzahl der Läsionen um weniger als $75 \%$ verringert hat (22) und jene, bei denen die Anzahl der Läsionen gestiegen ist (0).

Bei der Behandlung von Aktinischen Keratosen am Stamm und an den Extremitäten mit Ingenolmebutat 0,05\% erreichten 34,1\% der Patienten eine komplette Remission. Nach 12 Monaten Nachbeobachtungszeit blieben 85,1\% der Patienten mit kompletten Remissionen rezidiv frei [12].

Durch die Anwendung von Ingenolmebutat-Gel in der Praxis wurde bei $52 \%$ der Patienten, ohne Berücksichtigung der Reströtung sogar in 67,2\% der Behandlungen, eine komplette Remission nach 12 Wochen erreicht. Die Behandlung von Aktinischen Keratosen im Kopf-Hals-Bereich führte in 54\% zu kompletten Remissionen. Bei Anwendung am Stamm oder den Extremitäten konnten 38\% komplette Remissionen erzielt werden.

Die Auszählung der Läsionen ergab, dass bei 59\% der Patienten 12 Wochen nach Beginn der Therapie keine Läsionen vorhanden waren. Dieser Wert ist höher als der Anteil der kompletten Remissionen, errechnet über die Fläche. Einige Patienten wiesen zu diesem Zeitpunkt lediglich eine diffuse Rötung auf, ohne dass Läsionen erkennbar waren.

Wie in der Literatur beschrieben, sprachen mehr Aktinische Keratosen im Bereich Kopf/Hals (CR: 54\%; 30 von 58) als an

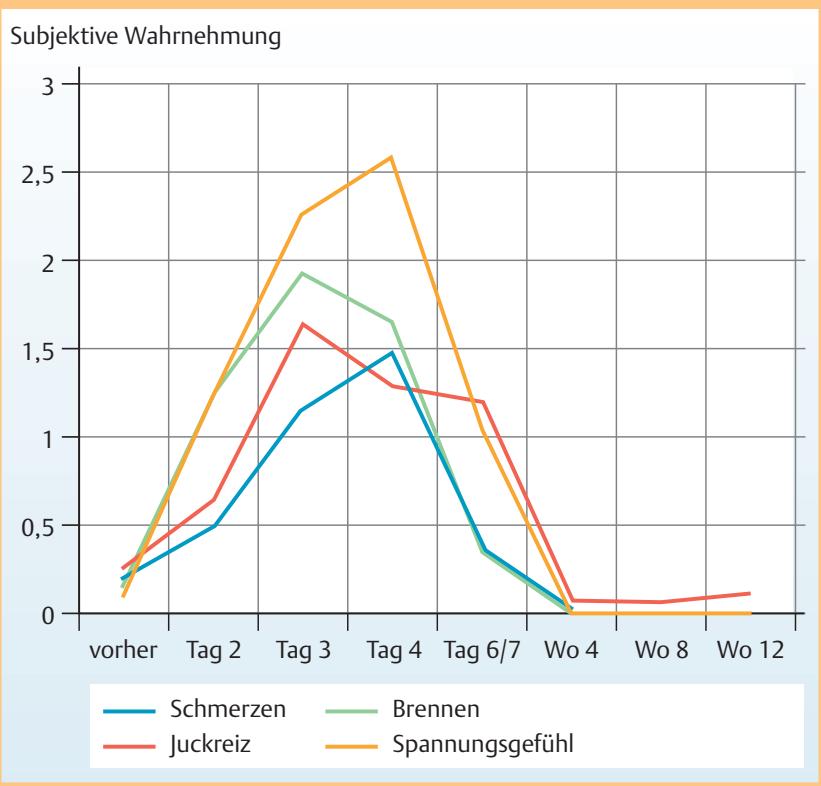

Abb.10 Entwicklung des subjektiven Wahrnehmens der Therapie im Verlauf der Behandlung. Bewertung der Merkmale Schmerzen, Juckreiz, Brennen und Spannungsgefühl in einer Skala von 0 bis $10 \mathrm{im}$ Verlauf der Behandlung (vorher: $n=63 ; \operatorname{Tag} 2: n=63 ; \operatorname{Tag} 3: n=63 ; \operatorname{Tag} 4: n=52$; Tag 6/7: $n=62$; Woche 4: $n=59$; Woche 8: $n=52$; Woche 12: $n=56$ ).

Stamm und Extremitäten (38\%; 3 von 8) auf die Behandlung an. Noch nicht untersucht ist das Ansprechen der Läsionen in Abhängigkeit der Lokalisation im Kopf-Hals-Bereich und des Geschlechtes. Es hat sich gezeigt, dass mehr Läsionen im unteren Gesichtsbereich (Wange: 63\% CR; Jochbein 58\% CR; Nase: 55\% CR) abheilten als auf dem Oberkopf (46\% CR) und der Stirn (45\%). Mit 64\% kompletten Remissionen zeigen weibliche Patienten eine höhere Ansprechrate auf die Therapie als männliche Patienten (42\% CR). Der Anteil männlicher Patienten mit einem progressiven Krankheitsverlauf (15\% PD) ist wesentlich höher als der weiblicher Patienten (4\%). Ob es hierfür eine kausale Erklärung gibt, gilt es noch zu untersuchen.

Verglichen mit den Ergebnissen der Zulassungsstudie konnte eine höhere Zahl an kompletten Remissionen erzielt werden. Eine mögliche Ursache liegt in dem kürzeren Beobachtungszeitraum von 57 Tagen in der Zulassungsstudie gegenüber den $91 \mathrm{Ta}$

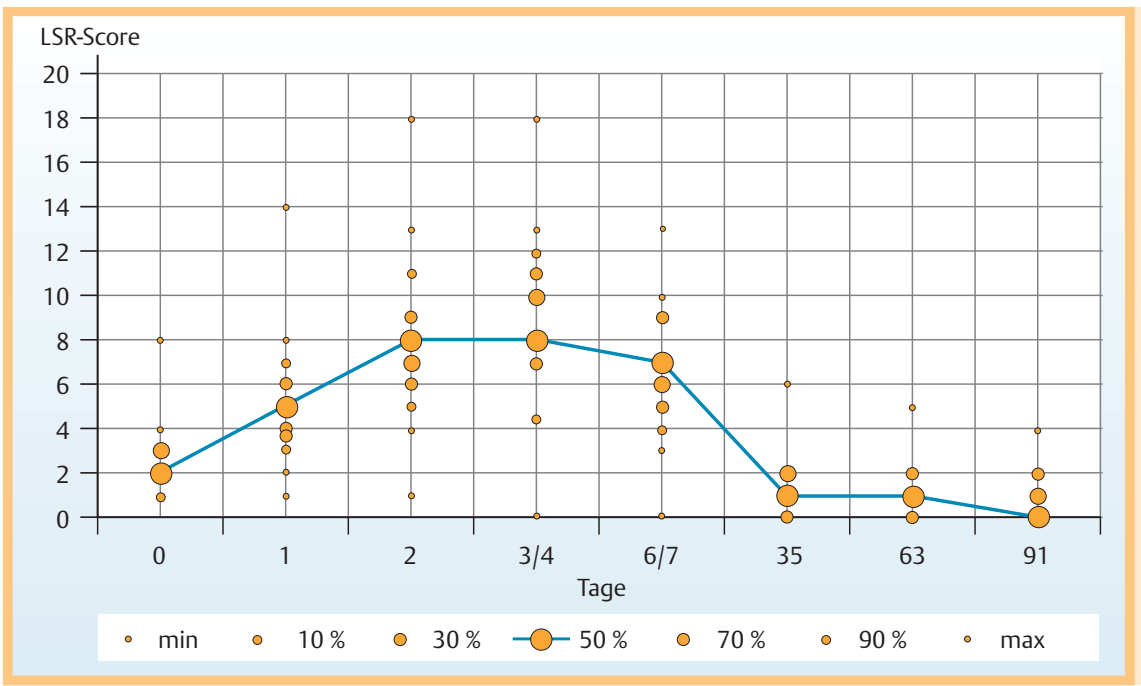

Abb.9 Local-Skin-Response-Score. Summe der Punktzahlen von 6 Merkmalen (Erythem, Schuppung, Krustenbildung, Ödembildung, Bläschenbildung, Erosionen und Ulzerationen), denen jeweils 0 bis 4 Punkte abhängig von der qualitativen Ausprägung des Merkmals zugeordnet werden. 
gen, nach denen in dieser Beobachtung der Therapieerfolg bewertet wurde. Ebenfalls konnte ein besseres Ansprechen von Aktinischen Keratosen im Kopf-Hals-Bereich als an Stamm und den Extremitäten beobachtet werden. Patienten aus besagter Zulassungsstudie zu Ingenolmebutat wurden stets auf einer Fläche von $25 \mathrm{~cm}^{2}$ mit Ingenolmebutat behandelt. Die Patienten dieser Publikation wurden abhängig von der Größe ihrer Läsionen therapiert $\left(11,4 \mathrm{~cm}^{2}\right.$; SD $\left.15,5 \mathrm{~cm}^{2}\right)$. Ungenauigkeiten in der Wirkstoffanpassung an kleinere Flächen stellen eine weitere mögliche Ursache für die höhere Rate kompletter Remissionen dar. Langzeitdaten aus der Praxis konnten aufgrund der kurzen Verfügbarkeit von Ingenolmebutat in Deutschland noch nicht erhoben werden.

Damit stellt diese Therapie eine vergleichbar effektive, wenn nicht sogar effektivere Möglichkeit der Behandlung von Aktinischen Keratosen dar, verglichen mit den aktuell etablierten Medikamenten.

Ein wesentlicher Vorteil von Ingenolmebutat ergibt sich aus der einfachen Handhabung. Während Diclofenac-/HyaluronsäureGel 2-mal täglich für bis zu 90 Tage und Imiquimod 5\% in bis zu 2 Zyklen 3-mal wöchentlich für 4 Wochen mit intermittierender 4-wöchiger Therapiepause aufgetragen wird, findet die Behandlung mit Ingenolmebutat-Gel lediglich einmal täglich an 2 oder 3 aufeinanderfolgenden Tagen statt. Dies stellt nicht nur eine deutlich angenehmere Behandlung dar, sondern verringert auch Probleme, die durch mangelnde Compliance seitens der Patienten entstehen können.

Hautreaktionen, erfasst durch den Local-Skin-Response-Score, traten vor allem am Anfang der Therapie auf. Diese Änderungen resultieren aus dem Wirkmechanismus von Ingenolmebutat und zählen somit nicht direkt zu den unerwünschten Nebenwirkungen. Aufgrund der hohen Potenz und kurzen Behandlungsdauer von Ingenolmebutat tritt die stärkste Hautreaktion bereits an Tag 3 und 4 nach Beginn der Behandlung auf. Vor allem starke Erythembildung, Schuppung, Schwellung und etwas verzögert auch Krustenbildungen bestimmen das Hautbild, was sich in den ersten Tagen unangenehm verändert. Die subjektive Empfindung von Juckreiz und Brennen wurde im Schnitt am 3. Tag mit 1,6 von 10 und 1,9 von 10 Punkten, Schmerzen und Spannungsgefühl am 4.Tag mit 1,5 und 2,6 von 10 Punkten am stärksten angegeben. Somit wird die Therapie mit Ingenolmebutat-Gel trotz der starken Hautreaktion nicht als besonders unangenehm empfunden, auch wenn die genannten Merkmale im Vergleich zum Therapiebeginn alle deutlich ansteigen.

Abschließend lässt sich sagen, dass Ingenolmebutat eine effektive und nebenwirkungsarme Behandlung der Aktinischen Keratosen ermöglicht und durch die einfache Handhabung der Therapie eine gute Alternative zu den bereits etablierten Medikamenten in der ambulanten Medizin darstellt.

\section{Interessenkonflikt}

Der Autor gibt an, dass kein Interessenkonflikt besteht.

\section{Ingenolmebutat for Treatment of Actinic Keratosis in an Outpatient Setting}

Actinic keratosis is a frequent disease. It may transform into a squamous cell carcinoma. 2013 a new drug called Ingenol mebutate gel has been approved. The effectiveness and manageability in the daily ambulant treatment has not been reported.

An analysis of 62 patients treated with Ingenol mebutate gel has been investigated and analyzed. The lesions of 58 patients were examined before starting therapy, after 8 weeks and after 12 weeks using photographic documentation. The patients were questioned for afflictions during treatment.

After 12 weeks $51.7 \%$ (30 patients) demonstrated complete remission of their lesions. 3.4\% ( 2 patients) achieved $\geq 75 \%$ clearance. The patients specified little discomfort during treatment.

Ingenol mebutate gel has been applied to the skin for 3 consecutive days by the ambulance stuff. Ingenol mebutate gel is an effective, well-tolerated treatment for actinic keratosis in daily outpatient treatment.

\section{Literatur}

1 Ackerman AB, Mones JM. Solar (actinic) keratosis is squamous cell carcinoma. Br J Dermatol 2006; 155: 9-22

2 Trakatelli M, Ulrich C, del Marmol V et al. Epidemiology of nonmelanoma skin cancer (NMSC) in Europe: accurate and comparable data are needed for effective public health monitoring and interventions. $\mathrm{Br}$ J Dermatol 2007; $156: 1-7$

3 Lucas R, McMichael T, Smith W et al. Environmental Burden of Disease Series, No. 13. Solar Ultraviolet Radiation. Global Burden of Disease from Solar Ultraviolet Radiation. Geneva: World Health Organization, Public Health and the Environment, 2006

4 Memon AA, Tomenson JA, Bothwell J et al. Prevalence of solar damage and actinic keratosis in a Merseyside population. Br J Dermatol 2000; 142: $1154-1159$

5 Frost C, Williams G, Green A. High incidence and regression rates of solar keratoses in a queensland community. J Invest Dermatol 2000; 115: $273-277$

6 Stockfleth E, Ulrich C, Meyer T et al. Epithelial malignancies in organ transplant patients: clinical presentation and new methods of treatment. Recent Results Cancer Res 2002a160: 251 - 258

7 Glogau RG. The risk of progression to invasive disease. J Am Acad Dermatol 2000; 42: 23-24

8 Brash DE, Ziegler A, Jonason AS et al. Sunlight and sunburn in human skin cancer: p53, apoptosis, and tumor protection. J Invest Dermatol Symp Proc 1996; 1: 136-142

9 Schwartz RA, Bridges TM, Butani AK et al. Actinic keratosis: an occupational and environmental disorder. J Eur Acad Dermatol Venereol 2008; 22: 606-615

10 Anwar J, Wrone DA, Kimyai-Asadi A et al. The development of actinic keratosis into invasive squamous cell carcinoma: evidence and evolving classification schemes. Clin Dermatol 2004; 22: 189-196

11 Leitlinie zur Behandlung der aktinischen Keratosen C44.X, 2011. AWMF-Register Nr. 013/041 Klasse: S1

12 Lebwohl M, Swanson N, Anderson $L L$ et al. Ingenol Mebutate Gel for Actinic Keratosis. New England Journal of Medicine 2012; 366: $1010-1019$

13 Loven K, Stein L, Furst K et al. Evaluation of the efficacy and tolerability of $0.5 \%$ fluorouracil cream and $5 \%$ fluorouracil cream applied to each side of the face in patients with actinic keratosis. Clinical Therapeutics 2002; 24: 990-1000

14 Lebwohl M, Dinehart S, Whiting D et al. Imiquimod 5\% cream for the treatment of actinic keratosis: results from two phase III, randomized, double-blind, parallel group, vehicle-controlled trials. Journal of the American Academy of Dermatology 2004; 50: 714- 721

15 Fariba I, Ali A, Hossein S et al. Efficacy of 3\% diclofenac gel for the treatment of actinic keratoses: A randomized, double-blind, placebo controlled study. Indian Journal of Dermatology, Venereology and Leprology 2006; $72: 346$ 